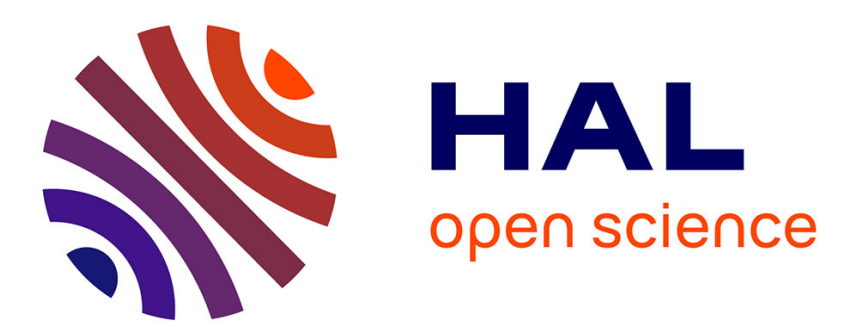

\title{
Relire les étymologies hébraïques à la lumière des étymologies arabes dans le Tesoro de la lengua castellana o espanola de Sebastian de Covarrubias (1611)
}

Dominique Neyrod

\section{- To cite this version:}

Dominique Neyrod. Relire les étymologies hébraïques à la lumière des étymologies arabes dans le Tesoro de la lengua castellana o espanola de Sebastian de Covarrubias (1611). reCHERches. Culture et Histoire dans l'Espace Roman, 2015, Langue, grammaire et didactique en diachronie: domaine roman (14), pp.69-84. hal-02043770

\author{
HAL Id: hal-02043770 \\ https://hal.science/hal-02043770
}

Submitted on 21 Feb 2019

HAL is a multi-disciplinary open access archive for the deposit and dissemination of scientific research documents, whether they are published or not. The documents may come from teaching and research institutions in France or abroad, or from public or private research centers.
L'archive ouverte pluridisciplinaire HAL, est destinée au dépôt et à la diffusion de documents scientifiques de niveau recherche, publiés ou non, émanant des établissements d'enseignement et de recherche français ou étrangers, des laboratoires publics ou privés. 


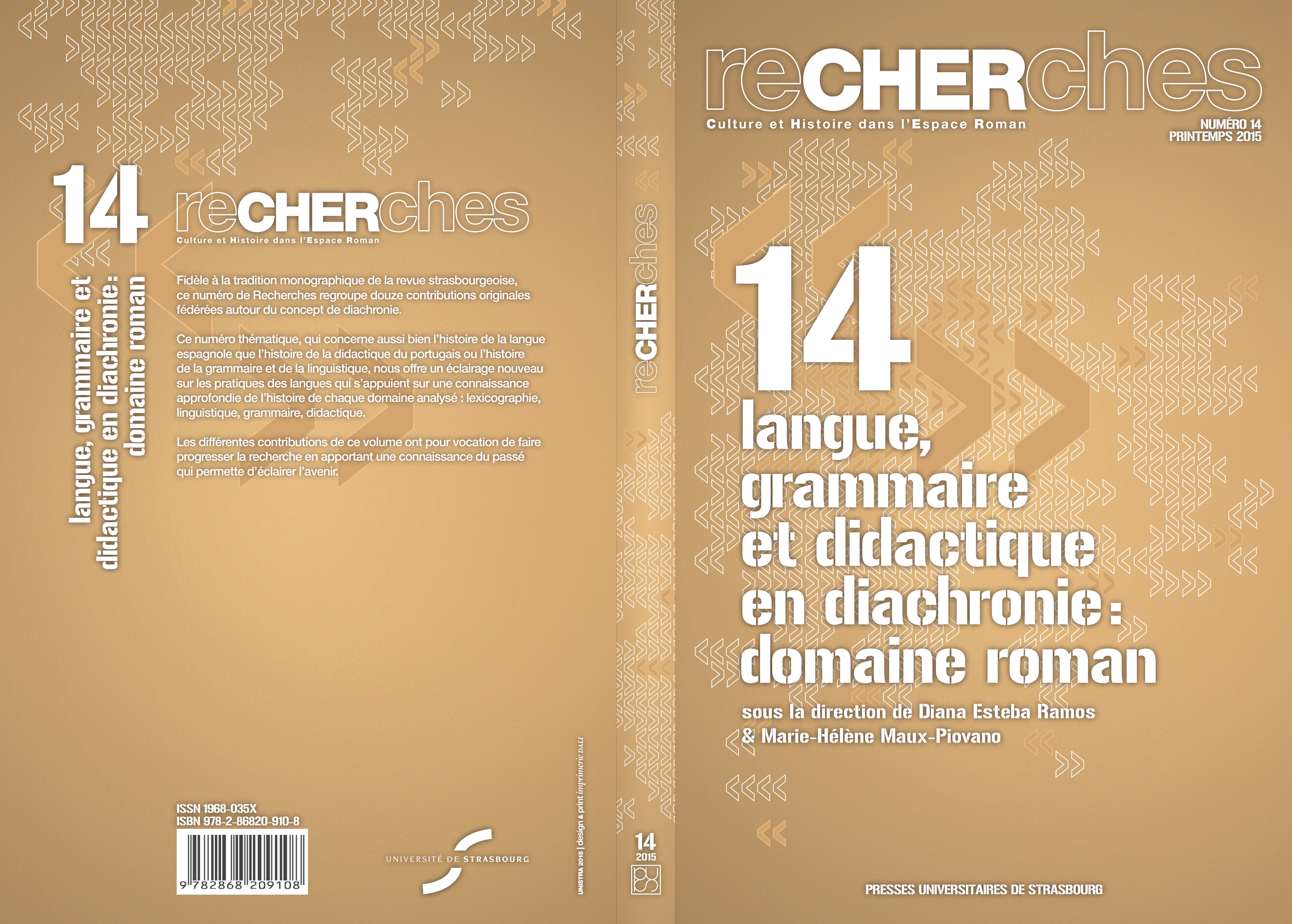




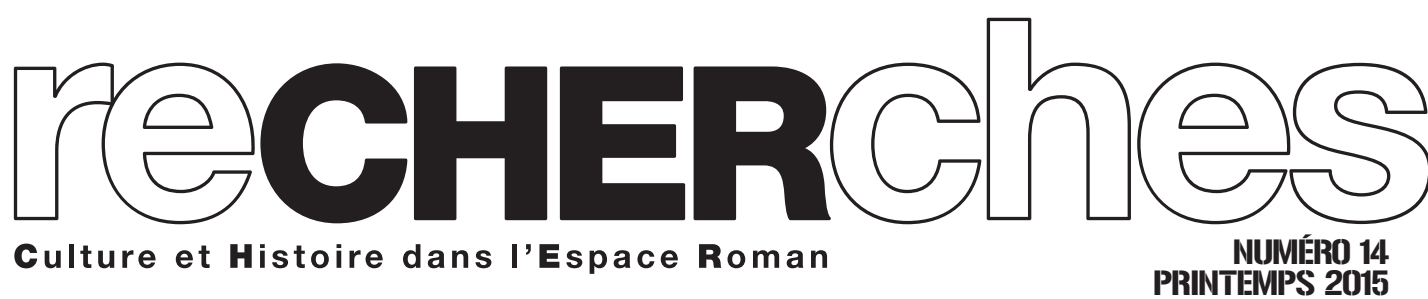

Culture et Histoire dans l'Espace Roman

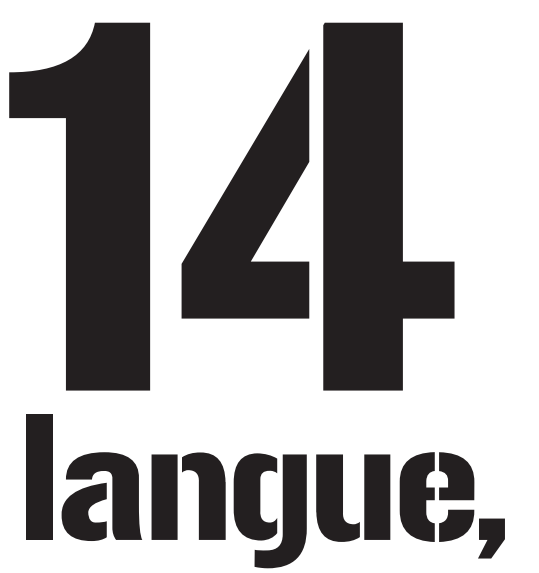
cyrammaire e't diclacticulue en diachronie: comaine roman

sous la direction de Diana Esteba Ramos

\& Marie-Hélène Maux-Piovano 


\section{Sommaire}

\section{Diana Esteba Ramos et Marie-Hélène Maux-Piovano}

Avant-propos.

Christian Lagarde

Cent ans après Saussure, retour sur la langue comme «fait social»

Emiliano Battista y Esteban Lidgett

Clases de palabras y categorías gramaticales (1932): renovación de la tradición gramatical escolar en unas conferencias inéditas de Amado Alonso

José Antonio Vicente Lozano

La suerte diasistemática de un contacto lingüístico:

lenguas afroasiáticas y románicas en la Península Ibérica

Mihai Enăchescu

El reemplazo de arabismos por latinismos: el caso de albéitar

Dominique Neyrod

Relire les étymologies hébraïques à la lumière des étymologies arabes dans le

Tesoro de la lengua castellana o española de Sebastián de Covarrubias (1611)... 69

\section{Susanna Allés Torrent}

Latinismos, palabras patrimoniales y soluciones perifrásticas en la práctica traductora de finales del siglo xv: el caso de Alfonso de Palencia

\section{Cristina Tabernero}

"Palabras inconvenientes" en el español del Siglo de Oro:

los términos injuriosos desde la perspectiva lexicográfica

Sophie Sarrazin

Le traitement de la forme en -ría dans la Grammaire et observations de

la langue espagnolle recueillies \& mises en françois de César Oudin (1597)

\section{Sónia Duarte}

A perceção do castelhano no Antidoto da Língua Portugueza (1710)

de António de Mello da Fonseca

Carmen Quijada Van den Berghe

La enseñanza del castellano como L2 a vascoparlantes en el siglo XIX

Jocenilson Ribeiro et Vanice Sargentini

L'imagerie scolaire dans les manuels de langue portugaise

au Brésil (1967-2007): un panorama historico-descriptif..

\section{Christianne Benatti Rochebois}

O livro didático de FLE elaborado no Brasil

nos anos da ditadura militar. 



\title{
Relire les étymologies hébraïques à la lumière des étymologies arabes dans le Tesoro de la lengua castellana o española de Sebastián de Covarrubias (1611)
}

\author{
Dominique NeYrod \\ Université du Maine
}

\begin{abstract}
Dlusieurs études récentes qui interrogent la place "démesurée» de l'hébreu et des étymologies hébraïques dans le Tesoro de la lengua castellana o española interprètent ce fait à la lumière du contexte humaniste dans lequel le Tesoro a été élaboré et d'autres critères tels que la formation d'hébraïsant de Covarrubias et son origine judéo-converse.
\end{abstract}

En ce qui concerne le cas particulier des arabismes pourvus par le lexicographe d'une étymologie hébraïque, on note qu'il est toujours expliqué, dans ces études, par le biais de cette assertion répétée dans le Tesoro à la façon d'un leitmotiv: «La lengua arábiga es deduzida de la hebrea».

Or les étymologies arabes du Tesoro - en particulier celles qui sont attribuées à Diego de Urrea- constituent un ensemble très remarquable par son contenu linguistique, qui non seulement apporte un démenti à la déclaration citée plus haut, mais qui peut aussi projeter de nouveaux éclairages sur la question des étymologies hébraïques, en suggérant de l'aborder selon une perspective linguistique et non plus exclusivement idéologique.

Nous nous proposons ainsi dans ces pages de comparer étymologie arabe et étymologie hébraïque d'un même mot, de commenter quelques exemples de terminologie appartenant aux deux traditions grammaticales, 
arabe et hébraïque, et de rappeler le lien entre la tradition grammaticale hébraïque et la pratique de l'exégèse scripturaire. Notre thèse est que dans le Tesoro plusieurs perspectives indépendantes sont juxtaposées: celle de la tradition humaniste, celle de la tradition grammaticale arabe et celle de la tradition grammaticale et exégétique hébraïque.

\section{"La lengua arábiga es deduzida de la hebrea »}

Nous commencerons par montrer, à l'aide de quelques exemples, comment, dans les études auxquelles nous avons fait allusion, la fameuse assertion «la lengua arábiga es deduzida de la hebrea » est prise comme clé ultime de la lecture et de l'interprétation à la fois des étymologies hébraïques et des étymologies arabes.

Ainsi, Fórneas-Besteiro (1991) écrit:

Lo fundamental de su [= de Covarrubias] enfoque de los "hebraísmos" es su convicción reiteradamente afirmada de que el árabe es una corrupción del hebreo.

L'auteur place cette remarque dans le cadre plus large d'une conception linguistique générale qu'il prétend être celle du lexicographe en affirmant:

Puede resumirse la concepción lingüística básica de Covarrubias, en el campo concreto que ahora nos atañe, diciendo que, para él, grosso modo, y la larga distancia cronológica, el árabe es al hebreo lo que el romance al latín. Esto explica suficientemente su punto de partida como las aplicaciones que de él hace.

Perea Siller (2006) constate de son côté que

aunque tenga en mente el origen hebreo de la lengua árabe, en la práctica Covarrubias pone de manifiesto muchos más arabismos que hebraísmos et souligne que la recherche d'étymologies hébraïques pour les vocables castillans se trouve considérablement encouragée par la présence effective, dans cette langue, de nombreux arabismes. Insistant, comme les autres auteurs, sur le fait que Covarrubias reflète les idées linguistiques humanistes sur le statut de l'hébreu, langue-mère de toutes les autres langues, Perea Siller fait néanmoins également appel au contexte sociolinguistique en Espagne, considérant que

es fácil deducir que el rechazo de la consideración social del árabe condujera a los etimólogos a hacer hebreas las palabras españolas de origen semítico. 
Quant à Or Hasson (2010), il examine concrètement le contenu linguistique des étymologies arabes et hébraïques et commente à ce propos l'article "almohada" du Tesoro. Covarrubias y expose en premier lieu l'étymologie arabe, que Or Hasson juge "perfectamente correcta, con todas las explicaciones morfológicas necesarias». L'étymologie arabe de "almohada" est suivie d'une étymologie hébraïque que Hasson juge en revanche défectueuse, tant du point de vue phonétique que lexical et morphologique. Voyant en cela une manifestation de la perspective idéologique dans laquelle s'inscrivent, selon lui, les étymologies hébraïques du Tesoro, il affirme que "el lexicógrafo aspira apoyarse en la razón lingüística» mais que «en fin, lo que le importa es comprobar, lógica o ilógicamente, la supremacía "del hebreo, que es la primera raíz de todas las lenguas y particularmente de la arábiga", incluso a costa de contradecirse a sí mismo y de violar la razón morfológica tanto hebrea como árabe».

\section{Almohada : étymologie arabe et étymologie hébraïque}

Examinons à notre tour le passage concerné de l'article "almohada" du Tesoro ${ }^{1}$ :

Dize Diego de Urrea que en su terminación arábiga se dize mehaddetum, del nombre haddum, que sinifica mexilla; y por ser nombre local almohada, tiene la letra $\mathrm{M}$ o la partícula mo, que sinifica lugar, cosa sobre que está otra; y assi al-mo-haddetum, corrompido dezimos almohada.

Il faut lire cette étymologie dans la perspective de la grammaire de l'arabe classique dont Diego de Urrea était à son époque un spécialiste reconnu. Dans haddum on lira /hadd/ 'joue' $+/ \mathrm{u} /$ désinence casuelle $+/ \mathrm{n} /$ marque de l'indétermination ou de l'état absolu du nom, appelée tanwīn (= "fait d'ajouter un n") dans la terminologie arabe ${ }^{2}$; le verbe correspondant est Ihadda/ 'creuser des sillons, raviner', dont les trois consonnes radicales sont [HDD]. Dans mehad-detum, on reconnaitra en outre le suffixe du féminin /-at/ transcrit ici $>$-et $<$ et le préfixe transcrit $>$ me- $<$ que

1 Sauf indication contraire, nos citations du Tesoro suivent l'édition de Martín de Riquer (1943).

2 Les arabisants occidentaux ont récemment adapté ce terme sous la forme "nounation". Cette marque est bien en arabe classique une nasale dentale et la transcription du mot selon les critères actuels sera /haddun/. Sur la graphie $>\mathrm{m}<\mathrm{du}$ tanwìn dans le Tesoro, voir Neyrod D. (2012: 657). 
Covarrubias appelle 'letra M' ou 'partícula mo' et sur lequel nous allons nous attarder un peu.

Il faut savoir en effet qu'en arabe, un grand nombre de noms (substantifs, adjectifs et participes, selon la terminologie des langues latines), sont dérivés par /m-/ préfixé et vocalisé /u/, /a/ ou /i/, selon les cas: /u/ pour les participes actifs et passifs des verbes aux différentes formes dérivées; /a/ pour les noms de lieu et de temps; /i/ pour les noms d'instrument. Ce cadre, qui est celui de l'arabe classique, évolue en arabe andalou, où la vocalisation en /i/ disparaît au profit de /a/ (/mi-/ > /ma- $/)$, la vocalisation /a/ évoluant elle-même dans de nombreux cas vers $/ \mathrm{u} /(/ \mathrm{ma}-/>/ \mathrm{mu}-/)^{3}$. L'article 'almohada' du $D C E C H$ de Corominas apporte les précisions suivantes:

[...] en hispanoárabe coexistían muhádda [qui figure dans le Vocabulista in arabico de Raimon Martí ( $13^{\circ}$ s.) et le Vocabulista aravigo de Pedro de Alcalá (1505)] et mahádda [chez Pedro de Alcalá], que es la forma que debiera esperarse, dada la sustitución de $m i$ - por $m a$ - en este dialecto [ = arabe andalou].

Federico Corriente (1999) donne de son côté l'évolution suivante: «almohada: del and. almuxádda $<\mathrm{cl}$. mixaddah»4.

On peut donc reconnaître, avec Or Hasson, que cette étymologie s'explique dans le cadre de la morphologie de l'arabe classique et de son évolution en arabe andalou et nous avons vu qu'elle présente également d'intéressantes questions caractéristiques de phonétique et de terminologie grammaticale. On est loin de l'assertion: «La lengua arábiga es deduzida de la hebrea».

Examinons maintenant l'étymologie hébraïque proposée par Covarrubias:

Sin embargo desto, digo que puede ser nombre hebreo, del verbo מעד, mahad, que sinifica declinare, reclinare, y sobre la almohada reclinamos la cabeça [...].

L'étymologie hébraïque, fait remarquer Or Hasson, repose sur deux types de similitude entre le vocable castillan 'almohada' et le vocable hébreu מעד. La première, qui est une similitude phonique, est récusée en vertu du fait que « esta semejanza existe solamente en el hebreo y el árabe castellanizados, en los cuales la $h$ puede reemplazar cuatro consonantes distintos, aleph, hé

3 Voir à ce sujet F. Corriente 1977: 78.

4 Le symbole $\mathrm{x}$ équivaut à $\mathrm{h}$ : nous respectons les symboles de transcription employés par chaque auteur. Mixaddah est un nom instrumental. Il est interprété comme nom local par Covarrubias et/ou Diego de Urrea du fait de l'évolution en arabe andalou de la vocalisation du préfixe /m-/. Dans la forme mehaddetum du Tesoro il reste à expliquer les graphies $<\mathrm{e}>$ pour $/ \mathrm{i} /$ et /a/: nous renvoyons à ce sujet à Neyrod D. (2012: 658-659). 
[laryngales simples sourdes], het et 'ayin [laryngales emphatiques sourde et sonore] o sus equivalentes árabes $»^{5}$; la seconde, qui est sémantique, repose selon cet auteur sur une traduction incorrecte, מעד signifiant en réalité 'tomber'6. La troisième critique faite à cette étymologie est morphologique et Or Hasson l'exprime en ces termes:

Si el lexicógrafo puede explicar tan bien, apoyándose en Urrea, la función de las partículas árabes, bien sabe que la $m$ en 'almohada' no pertenece a la raíz. No obstante, la $m$ vuelve a aparecer en la raíz hebrea7.

De ce cas, qui est loin d'être isolé dans le Tesoro, Or Hasson conclut à la posture idéologique de Covarrubias comme nous l'avons mentionné précédemment. Nous explorerons quant à nous une autre piste pour l'interprétation de ces faits: celle des traditions grammaticales arabe et hébraïque, dont des éléments sont présents dans le Tesoro.

\section{La terminologie grammaticale arabe et hébraïque dans le Tesoro}

Précisons avant tout que la terminologie grammaticale hébraïque dans le Tesoro a été bien remarquée, ce qui n'est pas le cas de la terminologie grammaticale arabe, pourtant très présente et de différentes façons,

Dominique Reyre (1997), par exemple, commente la présence du terme "seva" (articles "chisme", "guiñar"), ainsi que Or Hasson (2010) (article "cero") qui relève aussi d'autres termes tels que taham (article "acento"), hiphil, nom de la forme factitive du verbe, (article "añagaza") et "dages" qui constitue même une entrée du Tesoro:

DAGES: Cerca de los hebreos es un punto en medio de las letras que le reciben, el cual parece que las punça y despierta para que se pronuncien con más fuerça; es en dos maneras, fuerte y leve.

ACENTO: [...] Los hebreos gramáticos llaman al acento טעמ taham, sapor, gustus, consilium, ratio, probatio apta rei constructio, sermo, tandem accentum; del verbo טעמ taham, gustare [...].

5 Il faut noter que Covarrubias n'est pas étranger à ces questions de phonétique et de transcription. Voir par exemple l'article "behetría": «[...] algunos quieren sea de raíz hebrea, del verbo התר, hatar, fodere [...]. Otros que del verbo עתר, atar, con la letra aiin, que en la conjugación hiphil vale multiplicare verba». Le lexicographe s'efforce de transcrire différemment la spirante $h e($ par $>\mathrm{h}<$ ) et la pharyngale sonore 'ayin (par ø).

6 מעד = "fléchir" (Reymond 1991: 219).

7 Comme en arabe, le $(m e m / \mathrm{m} /)$ hébreu peut être un préfixe dans des séries nominales où il est vocalisé $/ \mathrm{a} / \mathrm{ou} / \mathrm{i} /$ (schèmes $\mathrm{MaCCaC}$ et $\mathrm{MiCCaC}$, principalement des noms de lieux, et MaCCeC, noms d'instrument et noms abstraits). Voir Jean, Ch-F. (1950: 66). 
AÑAGAZA : [...] Puede ser nombre hebreo, derivado del verbo נגש, nagas, que en hiphil vale facere accedere [...]

CERO: [...] Esta figura tienen en su cuenta guarisma los arábigos y la llaman çucum, que tiene en la escritura el oficio y valor que el seva cerca de los hebreos, que es de retraer la consonante a la sílaba que precedió.

CHISME: [...] Otros dizen ser nombre arábigo y averse dicho de gisme, chisme y chismoso; porque la gisme es una cierta señal, como una $\mathrm{O}$ pequeña, que los arábigos ponen sobre la letra $[q u e]^{8}$ retrahen a la precedente, y tiene la fuerça que el seva cerca de los hebreos, que son dos puntos uno debaxo del otro, que se ponen debaxo de la letra que se retrahe $[\ldots]$.

GUIÑAR: [...] Es nombre arábigo, y trae origen de la palabra gizme, que es cierta señal que los arábigos ponen encima de la letra que se ha de retirar con la precedente; y como los hebreos ponen debaxo de la dicha letra dos puntillos uno sobre otro, los arábigos ponen encima una o [...].

Nous observerons quant à nous que s'il apparaît bien à travers ces définitions que les termes dages, hiphil, taham sont spécifiques à la grammaire hébraïque et renvoient à des réalités qui lui sont propres, le terme seva par contre est le plus souvent associé par Covarrubias aux termes arabes çucum et gizme, qui renvoient à la même réalité, à savoir la fermeture syllabique. En effet, Covarrubias fait ici allusion au shewa dit "quiescent", signe orthographique indiquant en hébreu la fermeture de syllabe, tout comme le sukūn est en arabe un signe circulaire surmontant la consonne non vocalisée qui ferme la syllabe; quant au terme arabe jizm (de jazama "couper", "trancher") il désigne ce que nous appelons "apocope", marquée graphiquement par le sukūn. En vérité, si l’on se penche sur les articles "cero", "chisme", ou "guiñar", on constate que c'est bien la terminologie arabe qui est discutée, la terminologie hébraïque n'étant là qu'à titre de comparaison.

Il existe d'autres articles du Tesoro où apparaissent ainsi des termes propres à la grammaire arabe ou hébraïque. Plus intéressants à nos yeux sont les termes ou expressions terminologiques qui représentent des traductions-adaptations castillanes de la terminologie de ces deux traditions grammaticales. Par exemple, il faut reconnaître dans l'expression "en su terminación arábiga" qui figure toujours dans les étymologies attribuées à Diego de Urrea, un essai de traduction

8 Nous corrigeons une erreur de l'éditeur, Martín de Riquer, qui lit: «la gisme es una cierta señal, como una $\mathrm{O}$ pequeña, que los arábigos ponen sobre la letra $\mathrm{Q}$; retrahen a la precedente, etc.». L'édition de Arellano y Zafra ne corrige pas cette erreur. 
terminologique d'un concept-clé de la grammaire de l'arabe classique, ' $i$ 'râb, à la fois dans son sens usuel "donner un aspect arabe", "dire en (bon) arabe" et dans son sens technique "placer correctement les voyelles désinencielles". Et dans la phrase «tiene la letra M o la partícula mo» (voir "almohada"), il faut lire sous les termes "letra" et "partícula" un seul et même terme grammatical arabe harf, qui constitue la troisième catégorie de mots dans la classification opérée par la tradition grammaticale arabe, à côté des catégories du nom et du verbe ${ }^{9}$.

\section{Letra radical, letra aditicia, letra servil}

C'est sur les expressions "letra radical", "letra aditicia" et "letra servil" que nous nous arrêterons ici. Elles figurent abondamment dans les étymologies arabes et hébraïques du Tesoro, comme par exemple à l'article "almadena":

Hase de advertir que la $M$ que haze la silaba $m a$, en alma-dena, no es letra radical, sino servil y aditicia, que se pone en los nombres instrumentales, assí en la lengua arábiga como en la hebrea.

L'expression "letra aditicia" reflète la terminologie grammaticale arabe. C'est une lettre (harf) appartenant au groupe des hurüfaz-ziyāda, littéralement "lettres d'augmentation ou d'addition", qui sont des segments consonantiques n'appartenant pas à la racine du mot considéré, et qui révèlent ainsi le schème morphologique qu'il présente. La grammaire arabe compte dix hurüf susceptibles d'être des augments.

L'expression "letra servil" renvoie quant à elle à la tradition hébraïque qui divise les 22 lettres de l'alphabet en 2 groupes à peu près égaux, sujets à quelques variations selon les auteurs: 9 melakhim "rois" et 13 meshartim "serviteurs" dans la classification du grammairien du $x^{\mathrm{e}}$ siècle Dunash Ibn Labrat, mais 11 radicales et 11 serviles selon Ibn Djanāḥ ${ }^{10}$ (1889: 39). Les melakhim, écrit Cassuto (2007), "ne peuvent jamais être autre chose qu'un constituant de la base de l'entrée lexicale», alors que les meshartim,

9 Sur la terminologie grammaticale arabe dans le Tesoro, voir Neyrod D.: «Presencia de la terminología gramatical árabe en el Tesoro de la lengua castellana o española de Sebastián de Covarrubias (1611)». Communication présentée au IX Congreso de la SEHL, Córdoba, 11-13 septembre 2013.

10 «Les lettres essentiellement radicales» écrit-il «sont: גדזחטסעפצקר [gimel, dalet, zayin, het, tet, samekh, ayin, pe, tsadé, qof, resh], les autres lettres sont serviles, savoir: אבהויכלמנשת [aleph, bet, he, vav, yod, caf, lamed, mem, nun, shin, tav] qui s'ajoutent aux noms et aux verbes» (1889: 39). 
"lettres servantes ou serviles", "peuvent être soit un constituant, soit une lettre ajoutée pour des raisons grammaticales». C'est le cas, comme nous l'avons vu plus haut, du mem, préformante en hébreu de nombreuses formations nominales, ainsi que du mim, préformante en arabe des noms de lieu, des noms d'instrument et de la plus grande partie des participes actifs et passifs. C'est aussi le cas du he de l'hébreu en position d'article défini, comme le signale Covarrubias à l'article "haragán":

Es nombre arábigo, de raíz hebrea, del verbo רג, ragan, murmurare; cosa propia de ruines criados o peones y jornaleros reçongones [...]. Desta misma raíz se dixo harón, que vale floxo y tardo; bestia harona. Es de advertir que la $\mathrm{H}$ no es la letra radical en la una ni en la otra dicción.

D’autres termes ont aussi été employés pour désigner ces deux groupes et il est intéressant de noter que Sa 'adia Ga'on, grammairien juif oriental de la $1^{\text {re }}$ moitié du $\mathrm{x}^{\mathrm{e}}$ siècle, surnommé 'le père de la grammaire', nommait les melakhim "yesodot", "bases", et les meshartim "tosafot", "suppléments", s'inspirant ainsi pour ce dernier, semble-t-il, de la terminologie arabe.

Il faut savoir à ce propos que la majorité des grammairiens juifs, autant en Orient qu'en Espagne, écrivaient leurs traités en arabe, ce qui bien sûr favorise les emprunts et les calques terminologiques. Ils s'inspiraient également, pour leurs analyses linguistiques de l'hébreu, de la tradition grammaticale arabe, laquelle avait commencé à se former dès la fin du viır ${ }^{e}$ siècle avec le Kitāb de Sībawayhi. A ce sujet, Gonzalo Maeso (2001: 268) cite par exemple Mošé Ibn 'Ezra (1055-1135), poète et grammairien né à Grenade :

los israelitas que había en Al-Andalus, después de cierto tiempo, poco a poco, comprendieron los gustos de los árabes, y, gracias al esfuerzo desarrollado, entendieron su lengua, descollaron en ella, reflexionaron en la sutileza de sus dichos, se familiarizaron con el verdadero sentido de sus flexiones gramaticales [...]. Por ese camino Dios les reveló los misterios de la lengua hebrea y de su gramática, de las letras quiescentes, de la transformación, moción, reposo, permutación, absorción y otros fenómenos gramaticales, que captaron prontamente, comprendiendo de este modo lo que por tanto tiempo habían ignorado.

L'un des aspects les plus importants de ce processus d'imitation est "l'invention", dans la seconde moitié du $\mathrm{x}^{\mathrm{e}}$ siècle, par le grammairien juif Yehuda ben David Hayyuj (connu aussi sous son nom arabe Yahyā ibn Dāwūd, né à Fez et ayant vécu la majeure partie de sa vie à Cordoue), de la trilitéralité de la racine en hébreu, à l'instar de la racine arabe. 
Cela nous amène au terme "letra radical" et aux débats parmi les sémitisants autour de la question de la trilitéralité ou de la bilitéralité des racines consonantiques de l'arabe et de l'hébreu, voire de leur unilitéralité. Ce n'est pas ici le lieu pour développer cette question très controversée et je me contenterai de préciser que selon les spécialistes, le principe de trilitéralité de la racine consonantique est moins affirmé en hébreu qu'en arabe: «En arabe, la notion de racine trilitère était assez clairement définie ${ }^{11}$. En hébreu, la procédure d'extraction de la racine se montrait souvent beaucoup plus difficile ${ }^{12}$, d'où la valeur bien connue des travaux de Ben David Hayyudj al-Fasī» (Porkhomovsky 2007: 45). Cassuto (2007: 15-43) souligne que, avant Ben David Hayyuj et Ibn Djanāḥ ${ }^{13}$, «seule la stabilité des lettres dans les différentes formes importe. Ainsi, une base peut avoir une seule lettre, deux, trois ou bien plus, si ces lettres sont stables dans toutes les occurrences. Les lettres stables sont appelées "rois", les autres étant des "serviteurs" de ces dernières".

La théorie de la trilitéralité de la racine verbale, unanimement admise chez les grammairiens de l'hébreu à la Renaissance ${ }^{14}$, se reflète dans le Tesoro, (par exemple à l'article "corso": «[...] Es hebreo כרת [carat] es cortar propiamente y assí tiene todas tres letras radicales»), ainsi que l'opposition entre lettres radicales et lettres serviles. Quant à l'utilisation faite de ces outils d'analyse morphologique dans le Tesoro, elle diffère considérablement selon que l'analyse est celle de Diego de Urrea pour l'arabe ou celle de Covarrubias pour l'hébreu. Nous en avons vu plus haut un exemple avec "almohada", nous allons en voir un autre avec "almártega":

11 Selon Carter, M. G. (2004: 100-102), Sïbawayhi (c. 752- c. 796) s'appuyait sur le principe que le mot était le résultat de la combinaison de ses consonnes radicales dont le nombre pouvait aller de 1 à 5 , mais était le plus souvent de 3 , et d'un schème morphologique incluant les voyelles et éventuellement des augments consonantiques.

12 Kukenheim, Louis (1951: 106, n. 1) évoque l'œuvre du «professeur J. L. Palache [qui selon lui] prouve que, dans bien des cas, la bilittéralité de la racine constitue l'élément vivant dans l'esprit de ceux qui parlent, tandis que la base trilitère est toute théorique et abstraite: certaines racines apparaissent et ont toujours apparu comme des racines de deux et même d'une lettre».

13 «Les racines des verbes ont au moins trois lettres et, sans augment, au plus quatre» écrit Ibn Djanāh. Il en va différemment pour les noms: "La racine des noms simples est de deux lettres au moins [...] et de cinq lettres au plus [...]» $(1889: 36-37)$.

14 Voir García Jalón, S. (1998: 121) : «Entre los autores del XVI es general la convicción de que el verbo hebreo es por naturaleza trirradical. [...]. A partir del De rudimentis [de Iohannes Reuchlin, publié en 1506] todos los gramáticos europeos convienen en la trirradicalidad del verbo hebreo». 
Dize Urrea que en su terminación arábiga es mertacun, del verbo reteca, que sinifica tener fuerte y tirar para sí. Y porque lo reduzgamos a términos que todos lo puedan entender, señalémoslo así: al-m-reteca; la al es artículo, la $m$ es instrumental, por ser la almártega instrumento para retener el caballo, el reteca pierde la $e$, y retírase con la ma, y dezimos almárteca; la $\mathrm{C}$ y la $\mathrm{G}$ son letras permutables, tenue en media. Tras esto es de saber que el vocablo almártega es hebreo, del nombre רתק, ratach, que vale catena, por ser como cadena con que está preso el caballo y atado, de manera que con el artículo y la $\mathrm{M}$ dirá al-martach y de allí almártaga, conforme a lo que está dicho de la permutación de $\mathrm{C}$ en $\mathrm{G}$. A los que no supieren la lengua hebrea les ha de parecer todo algaravía, así el arábigo como el hebreo; los que tienen noticia de la lengua Santa me defenderán.

Diego de Urrea fait de mertacun l'étymon arabe de “almártega" et l'analyse correctement du point de vue morphologique comme nom instrumental dérivé par le préfixe /m-/ d'un verbe reteca ${ }^{15}$. L'étymologie hébraïque, elle, emprunte d'autres voies: elle ne part pas d'un verbe mais d'un nom, ratach "chaîne"16, auquel est préfixé un /m-/ qui n'a plus la fonction morphologique ni la valeur sémantique qu'il avait dans l'étymologie arabe. En fait, comme pour "almohada", cette étymologie repose sur une analogie sonore coïncidant avec une analogie sémantique. Quant au /m/ initial, nous nous demandons s'il n'a pas, dans ce cadre, une valeur simplement catégorielle, nous appuyant pour ce faire sur cette indication de García-Jalón (1998: 114) : " En la Gramática hebrea del XVI se denomina "hemánticas" o "heemánticas" a las letras אהמנתי [aleph, he, mem, nun, taw, yod], conjunto de consonantes que, por distintos motivos, puede añadirse a una formación trirradical. Son nombres "hemánticos"

15 Voir Corominas (DCECH, art. almártaga I) : «"especie de cabezada que se ponía a los caballos para tenerlos asidos cuando el jinete se apeaba", de una palabra hispanoár. * marta 'a íd., derivada de rátta' "trabar" (ár. clás. ráta' o 'arta "pacer libremente un animal en un prado lozano' [...]». «No es rara» ajoute l'auteur «la transcripción del 'árabe, africada glotal, por $g$, semioclusiva velar», et, comme le déclare Covarrubias, «la $c$ y la $g$ son letras permutables», de sorte que reteca pourrait parfaitement représenter l'ar. rátta'. Corriente (1999: 188) donne une étymologie similaire: «del and. *al-mártąa por cl. ritā̧ "traba del ganado", ambos n. instr. formados con metanálisis de raíz sobre el verbo cl. irta\}a "pacer" < $\{\mathrm{r}\{y\}$ » tout en signalant que ces formes (al-mártąa, ritā $\varphi$ ) sont le résultat d'une mauvaise analyse de la racine, à laquelle aurait été intégré le /t/ du verbe irtaৎa, qui a là statut d'augment et non de radicale.

16 D'après Reymond, il existe dans la Bible des formes $/ \mathrm{ratuq} /$, /ratuqah/, /ratic/, /retuqah/ usuellement traduites par "chaîne(s)" mais dont le sens est en réalité très incertain et la forme de base non-attestée (1991: 362). 
aquéllos en los que aparece una de esas letras ${ }^{17} »$. García-Jalón cite les grammaires de Ioannes Isaac (1570), Bellarmino (1596), Rapheleng (1572) qui mentionnent explicitement cette classe de mots et il ajoute que d'autres grammairiens, sans établir un type distinct en fonction de ces lettres recourent néanmoins à elles dans leurs classifications des formations nominales. Il précise également que «al estudiar ese tipo de formaciones nominales, no son tomados en consideración ninguno de los valores semánticos que esas letras pueden comportar ", à savoir, pour la préformante $/ \mathrm{m}-/$, noms de lieu, noms d'instrument, noms abstraits (v. note 6).

\section{Exégèse biblique et grammaire hébraïque}

Il faut tenir compte du fait que la part de la réflexion analogique et des phénomènes de transposition chez les grammairiens hébraïques médiévaux est considérable, et que l'un et l'autre sont des outils d'analyse grammaticale. "Il faut savoir qu'il y a deux espèces d'irrégularités» écrit Ibn Djanah " $1^{\circ}$ ce qui s'écarte de l'analogie, $2^{\circ}$ ce qui s'écarte de l'usage adopté par la catégorie, tout en étant conforme à l'analogie. C'est que l'usage existe en effet de deux façons; il est tantôt conforme à l'analogie et tantôt y est contraire, mais la conformité avec l'analogie est le cas le plus fréquent» (1889: 331). Ou encore: "Sache qu'il y a en hébreu deux espèces de transpositions: celle des lettres [= métathèse] et celle du sens [=hypallage]» (1889: 334). Ce cadre grammatical est aussi celui de l'exégèse scripturaire, deux disciplines inséparables: les plus grands noms de la grammaire hébraïque, tels que Menahem ben Saruq au $\mathrm{x}^{\mathrm{e}}$ siècle, Ibn Djanah au XI ${ }^{\mathrm{e}}$, les Qimhí, Joseph au XII ${ }^{\mathrm{e}}$ et ses fils Moïse et David aux XII ${ }^{e}$ et début du XIII ${ }^{\mathrm{e}}$, pour ne citer qu'eux, ont été aussi de grands exégètes. Au sujet de Ibn Djanah, Gonzalo Maeso écrit: «Las dos partes, gramática y léxico, que abarca su opus magnum, el Kitāb al-tanquih (Libro de la crítica) o investigación minuciosa del lenguaje [...] encierran una aportación extraordinaria a la exégesis bíblica» (2001: 145), insistant plus loin sur son «Kitāb al-ūsūl (Libro de las raíces), diccionario hebreo

17 Campensis précise cependant explicitement que aleph, he, mem, nun, taw, yod permettent des dérivations nominales à partir des verbes (souligné par nous): «De litteris האמנת. Sex sunt litterae per quas fiunt derivationes nominum a verbis [...]» (cité par Kukenheim L. 1951: 105, n. 3). Ibn Djanāḥ, lui, insiste sur l'analogie sémantique entre les bases et leurs dérivés: «Le mêm se met au commencement des noms (participes) qui pour le sens tiennent des mots dont ils dérivent» (1889: 55). 
etimológico, con multitud de textos de la Escritura, con lo cual resulta también, indirectamente, un manual de exégesis bíblica» (2001: 265). Et à propos des Qimhí, il ajoute: "Los tres realizaron, aparte de sus obras gramaticales y lexicológicas, valiosos comentarios bíblicos sobre el Pentateuco, Proverbios, libro de Job [...]» (2001: 148).

Les grammairiens hébraïsants occidentaux sont héritiers de cette tradition. Après Ibn Djanāḥ, écrit Kukenheim, "nous en arrivons aux sources mêmes des hébraïsants de la Renaissance, à savoir Ibn Esra (XIree siècle) à qui nous devons nos connaissances des premiers grammairiens [...] et les Kimchi [=Qimhíi] (XII ${ }^{\mathrm{e}}$ siècle) [...]. Ce sont là les auteurs que Reuchlin cite comme ses maîtres» (1951: 93). La transmission se fait directement ou à travers des traductions en latin d'œuvres grammaticales (de Moïse Qimhí par exemple, traduite par Sébastien Munster) ou exégétiques (de David Qimhí, traduites par Alfonso de Zamora, juif convers né en 1474, qui enseigna l'hébreu dans les universités d'Alcalá et de Salamanque dans la première moitié du Xvi ${ }^{\mathrm{e}}$ siècle et fut l'auteur d'une grammaire hébraïque en latin et de nombreux travaux lexicologiques (Gonzalo Maeso 2001: 270-271) ${ }^{18}$.

Cette tradition se poursuit avec les différents professeurs qui vont se succéder à la chaire d'hébreu de l'Université de Salamanque et, comme l'a exposé Dominique Reyre dans plusieurs articles très documentés (1997, 1999, 2006), Covarrubias, qui a étudié l'hébreu dans cette université entre 1565 et 1571, applique dans son effort étymologique les techniques de l'exégèse scripturaire enseignée par ses maîtres.

L'étymologie de "halago", par exemple, qu'il attribue à l'hébraïsant Pedro de Palencia nous fournit quelques indications sur cette approche:

Vale caricia engañosa, quasi falago a fallendo. Halagar, acariciar, asegurar y amansar. A la gala, como הלק, khalaqh, es halagar, es alisar el cabello y es amansar a uno; de ay es la $p$ en $\lambda$, halagar. Esto es del padre fray Pedro de Palencia.

On observe que le recours à l'analogie sonore y est explicite - la gala, como הלק, khalaqh - et s'enrichit d'une explication basée sur la motivation sonore du signifiant, le passage de la sourde $/ \mathrm{q} /$, p , à la sonore $/ \mathrm{g} /$, ג, étant mis en rapport avec la signification "amansar". Précisons que l'hébreu biblique connait de nombreuses formations verbales et nominales sur חלק (avec het et non he initiale), "être glissant>être trompeur", au nombre

18 Sur ce sujet, v. aussi Kukenheim (1951: 88-93) 
desquelles חָ̣ halaq, "fausseté, flatterie" (Reymond 1991:129-130). Corriente qui recueille "halagar" dans son Diccionario de arabismos rejette pour différentes raisons strictement linguistiques l'étymologie ar. cl. xālaq "tratar amablemente", proche morphologiquement et sémantiquement de l'hypothèse hébraïque du Tesoro. On notera par ailleurs que de nombreuses dérivations romanes de $h /$ falagar abritent la notion de séduction (v. Corriente, 1999: 99).

Covarrubias présente également des étymologies hébraïques sans attribution et qui sont peut-être de sa fabrication. Nous prendrons l'exemple de "alguazil"19 où, après avoir rapporté l'étymologie arabe de Guadix et celle de Urrea, qui insiste sur le fait que «todos los nombres que empieçan gua los árabes los pronuncian por $v a$, con la l, vau, de los hebreos, pero los moriscos de España la pronuncian gua, de modo que por al vasil dizen al guazil», le lexicographe poursuit: "Sin perjuyzio de lo dicho, porque yo doy gran crédito a Diego de Urrea, podríamos dezir que algazil es hebreo, del verbo ג gaçal, rapere ${ }^{20}$, porque echa mano del delinqüente». Une telle proposition, outre qu'elle est basée sur l'analogie sonore et sémantique, recourt également au remplacement de la consonne radicale initiale /W/, lettre servile, par /G/, lettre radicale.

Il nous semble que cette intervention sur le signifiant peut être reliée, même indirectement, aux catégories de la permutation, transposition et interversion de lettres qui figurent chez Ibn Djanah ${ }^{21}$ ainsi qu'aux pratiques de la cabale que Dominique Reyre a relevées dans le Tesoro. Il s'agirait ici de la temura, "del hebreo mur que es mudar y consiste en la sustitución de unas letras por otras para descubrir significados ocultos» (Reyre 2006: 25) qui, avec le notaricon, est mis à l'œuvre par Covarrubias $^{22}$.

19 "Oficial inferior de justicia": del and. alwazír < cl. wazìr, una adaptación a la raíz $\{w z r\}$ del pahleví wičîr "consejero" (Corriente 1999: 102)

20 Voir Reymond (1991: 80) : גזל "prendre par violence" (= piller, arracher, enlever, s'emparer de) dans différents passages de la Bible.

21 Voir Ibn Djanāh, (1889: chap. VI, XXXI, XXXII).

22 Nous renvoyons pour ces questions à Reyre, 1997, 1999, 2006 et nous contentons d'ajouter que dans son De arte cabalistica (1517), Johann Reuchlin écrit que les trois parties de l'art cabalistique sont la Gymateria, le Notarikon et la Temura «qui sont l'arithmétique, l'art notatoire et la permutation des lettres» (1973: 259). Dans la temura, «il se fait un échange réciproque d'une lettre pour une lettre, et cela se produit autant de fois que les alphabets se permutent. Ces permutations se font selon le nombre des lettres, 22 fois, car l'alphabet hébreu a 22 lettres. [...]» (1973: 284). La temura est désignée dans le Tesoro par le terme "trastrueque de letras", écrit Reyre; 


\section{Conclusion}

Les étymologies hébraïques du Tesoro sont donc d'une grande complexité, à la mesure de la complexité du système grammaticoexégétique mis en place par les grammairiens hébreux du Moyen Âge et perpétué par les hébraïsants chrétiens et humanistes de la Renaissance.

Nous avons choisi de les aborder à la lumière des étymologies arabes afin de montrer que, en juxtaposant la perspective humaniste idéologique, la tradition grammaticale et exégétique hébraïque et la tradition grammaticale arabe ${ }^{23}$, Covarrubias se trouve au croisement de différents savoirs de son temps, certains plus anciens, d'autres plus modernes.

La modernité est représentée par les étymologies arabes fournies par Diego de Urrea à partir de l'arabe classique et souvent poursuivies et complétées dans une perspective évolutive vers l'arabe andalou puis vers le castillan ${ }^{24}$, qui se situent dans un cadre purement linguistique. La personne de Diego de Urrea, comme son travail étymologique, représentent les débuts de l'arabisme scientifique occidental ${ }^{25}$.

nous ajoutons que dans certains cas, cette expression et la permutation de lettres qu'elle désigne renvoient simplement à un processus attesté en phonétique, tel que l'interversion de liquides, comme dans "muladar": "y porque es fuera de los muros, se dixo muradal, y de alli muladar, trocando las letras».

Du notaricon («del arameo notar, que es indicar y consiste en utilizar las letras de una palabra como iniciales de otras») (Reyre, 2001,25) qui est désigné dans le Tesoro par l'expression "poner letra por parte", l'article "gafo" du Tesoro fournit un exemple impressionnant emprunté à Saint Jérôme. Reuchlin définit ainsi le notaricon: «C'est une certaine convention reçue secrètement chez les Cabalistes, que des lettres prises séparément désignent, sans assembler des lettres en syllabes, certains mots, comme ont l'habitude d'en user les secrétaires et les comptables, et comme parfois les chefs de guerre dans leurs correspondances secrètes, qui exprimaient les desseins cachés de leur esprit en usant de lettres replacées sans ordre». Et il poursuit: "[c'est comme] si d'aucuns étaient convenus de symboliser par la lettre Iod, à cause de sa figure de point indivisible, l'ineffable Tétragramme que nous interprétons couramment Seigneur [YHWH (yod, hé, vav, hé) = Yahvé]» (1973: 269-270).

23 Malgré l'influence de l'analyse grammaticale de l'arabe sur celle de l'hébreu, les deux traditions divergent et la part de l'exégèse dans la tradition grammaticale hébraïque s'explique en partie par le fait que «les Arabes avaient eu l'avantage de décrire une langue vivante, les Juifs devaient ressusciter une langue morte et dont, en outre, les traditions ne concordaient pas entre elles» Kukenheim (1951: 92).

24 Voir à ce sujet Neyrod D. (2012).

25 Sur Diego de Urrea, voir Rodríguez Mediano F. \& García-Arenal M. (2002) et Rodríguez Mediano F. (2004). Sur Diego de Urrea dans le Tesoro, v. Neyrod D. (2012). 
Quant à Covarrubias, malgré son adhésion proclamée à certains crédos du milieu humaniste auquel il appartient par sa formation ( «la lengua arábiga es deduzida de la hebrea», "el hebreo, primera raíz de todas las lenguas y particularmente de la arábiga»), on ne saurait voir dans ses étymologies hébraïques le seul désir de se conformer à cette idéologie, en faisant fi de toute rigueur scientifique, comme le déclare Or Hasson à propos de "almohada". Covarrubias met à l'épreuve sa formation d'hébraïsant en utilisant des outils spécifiques et il aboutit ce faisant à des propositions étymologiques qui ne peuvent être abordées que dans ce cadre de pensée.

\section{Bibliographie}

Carter Michael G., 2004, Sibawayhi, New Delhi, Oxford University Press.

Cassuto Philippe, «Base, roi et serviteur», La formation des mots dans les langues sémitiques, Cassuto Ph. \& Larcher P. (éd.), Langues et Langages 15, Université de Provence, p. 15-43.

Corominas Joan et Pascual José A., 1991, Diccionario crítico etimológico castellano e hispánico (DCECH), Madrid, Gredos.

Corriente Federico, 1999, Diccionario de arabismos y voces afines en iberorromance, Madrid, Gredos.

Corriente Federico, 1977, A gramatical sketch of the Spanish Arabic dialect bundle, Madrid, Instituto hispano-árabe de cultura.

Covarrubias Sebastián (de), 2003 [1943], Tesoro de la lengua castellana o española, Martín de Riquer (ed.), Barcelona, Alta Fulla.

Fórneas-Besteiro José María, 1991, «Los 'hebraísmos' del Tesoro de Covarrubias», Miscelánea de estudios árabes y hebraicos XXXVII-XXXVIII, Université de Granada, p. 223-247.

García-Jalón de la Lama Santiago, 1998, La gramática hebrea en Europa en el siglo XVI. Guía de lectura de las obras impresas, Salamanca, Publicaciones Universidad Pontificia.

Gonzalo Maeso David, 2001, El legado del judaísmo español, Madrid, Trotta.

Hasson Or, 2010, «"La lengua santa, fuente y principio de todas las demás". Sobre el estado privilegiado del hebreo en el Tesoro de la lengua castellana o española de Covarrubias» in: La biblia en el siglo de oro, Fine Ruth \& Arellano Ignacio (eds) Iberoamericana/Vervuert, p. 257-274.

Ibn Djanah, 1889, Le livre des parterres fleuris d'Abou'l-Walid Merwan ibn Djanah, traduit en français sur les manuscrits arabes par le rabbin Moïse Metzger, Paris, F. Vieweg, E. Bouillon.

Jean Charles-F., 1950, Grammaire hébraïque élémentaire, Paris, Letouzey et Ané. 
Kukenheim Louis, 1951, Contributions à l'histoire de la grammaire grecque, latine et hébraïque à l'époque de la Renaissance, Leiden, E. J. Brill.

Neyrod, Dominique, 2012, "Dize Diego de Urrea que... o los discursos entrelazados de Diego de Urrea y Sebastián de Covarrubias sobre palabras arábigas en el Tesoro de la lengua castellana o española» in: Historiografía lingüística: líneas actuales de investigación, Battaner Moro E., Calvo Fernández V., Peña Jiménez P. (eds), Münster, Nodus Publikationen, vol. II, p. 655-663. Perea Siller Francisco Javier, 2006, «En torno a la historia lingüística universal y española en Sebastián de Covarrubias", in: Studia linguistica et philologica in memoriam Feliciano Delgado, Ma Luisa Calero Vaquera, Fr. Osuna García, A. Zamorano Aguilar (eds), U. de Córdoba, p. 229-249.

Porkhomovsky Victor, 2007, «La structure de la racine et la formation des mots dans la tradition sémitologique russe» in: La formation des mots dans les langues sémitiques, Cassuto Ph. \& Larcher P. (éd.), Langues et Langages 15, Université de Provence, p. 45-52.

Reuchlin Johann, 1973, La Kabbale (de arte cabalistica), introduction et traduction par François Secret, Paris, Aubier Montaigne.

Reymond Philippe, 1991, Dictionnaire d'Hébreu et d'Araméen Bibliques, Les Editions du Cerf.

Reyre Dominique, 1997, "Cuando Covarrubias arrimaba el hebreo a su castellano», Criticón 69, p. 5-20.

Reyre Dominique, 1999, «Fray Luis de San Francisco, hebraísta cristiano del Siglo de Oro frente a la cábala rabínica», Criticón 75, p. 69-89.

Reyre Dominique, 2006, «La Biblia en el diccionario de Sebastián de Covarrubias. Cuestiones filológicas y exegéticas », Ínsula 108-109, p. 22-25

Rodríguez Mediano Fernando \& García-Arenal Mercedes, 2002, «Diego de Urrea y algún traductor más: en torno a las versiones de los 'Plomos' " Al-Qantara, vol. 23, p. 499-516.

Rodríguez Mediano Fernando, 2004, «Diego de Urrea en Italia» Al-Qantara, vol. 25 . 